\title{
SKLB1002, a novel inhibitor of VEGF receptor 2 signaling, induces vascular normalization to improve systemically administered chemotherapy efficacy
}

\author{
G. SHEN ${ }^{\ddagger}$, Y. LI", T. DU*, G. SHI, L. DAI, X. CHEN, R. ZHENG, W. LI, X. SU, S. ZHANG, Y. WEI, S. YANG, H. DENG* \\ State Key Laboratory of Biotherapy and CancerCenter, West China Hospital, Sichuan University, Chengdu, China
}

${ }^{*}$ Correspondence: denghongx@scu.edu.cn

${ }^{\ddagger}$ Contributed equally to this work.

Received February 7, 2012 / Accepted March 15, 2012

\begin{abstract}
Vascular endothelial growth factor receptor (VEGFR) or vascular endothelial growth factor (VEGF) inhibitors have shown only modest clinical activity for most tumor types when used as single agents. However, present evidence indicates that these antiangiogenic drugs can cause transient "normalization" of the tumor vasculature, thereby improving the delivery of systemic chemotherapy. We examined temporal changes in tumor vascular function in response to the novel VEGFR2 inhibitor, SKLB1002. Established tumor-bearing animals were evaluated at serial time points for treatment-associated changes in tumor vascular architecture and function. As a result, blocking VEGF signaling by SKLB1002 produced a morphologically and functionally "normalized" vascular network. Consistent with our observations, a 2.2 fold increase in intratumoral doxorubicin levels was determined with SKLB1002 pretreatment compared with administration of doxorubicin alone. Finally, combined SKLB1002 and doxorubicin exhibited significant antitumor (49\% of control size) and antimetastatic effects (12\% of control metastatic nodules) in vivo. Our results showed SKLB1002 induced vascular normalization and enhanced anticancer drug delivery, which were associated with the observed synergistic effect in vivo.
\end{abstract}

Key words: vascular normalization, chemotherapy, hypoxia, metastasis

Solid tumors account for about $91 \%$ of cancer mortality [1]. To obtain oxygen and other nutrients as well as an ability to evacuate metabolic wastes, solid tumors sprout new vessels from existing ones and recruit endothelial cells progenitors from the bone marrow. Angiogenesis is induced early during the multistage development of carcinogenesis and considered as one of the principal hallmarks of cancers [2]. Vascular endothelial growth factor (VEGF) and its receptor (VEGFR2) is the predominant mediator during tumor angiogenesis process. VEGF promotes endothelial cell proliferation, migration, survival. In addition, VEGF upregulation is associated with hypoxia and oncogene signaling including mutant ras and EGFR. Because of its fundamental functions in tumor angiogenesis, VEGF is recognized as a valuable target for antiangiogenic cancer therapy [3].

There are currently a number of notably antiangiogenic drugs targeting VEGF signaling in clinical use [4]. Unfortunately, these effects are often transitory and followed by a restoration of tumor growth and progression [5]. In contrast, when given in combination with chemotherapy, bevacizumab (a humanized anti-VEGF antibody) yielded significant improvement in survival among patients with metastatic colorectal cancer [6]. It is a persistent paradox that destroying the vasculature would severely reduce the delivery of oxygen and drugs to the solid tumor, rendering chemotherapeutics less effective.

Essentially, the abnormal vasculature of tumors and the resulting abnormal tumor microenvironment together form a formidable barrier to the delivery of drugs and oxygen. Recently, Jain and colleagues propose that antiangiogenic therapies can transiently result in a "normalization" of aberrant tumor vasculature. It involves the pruning and remodeling of the tumor vasculature, which is characterized by less tortuous vessels and tighter basement membrane. These morphological changes are accompanied by functional changes including reduced interstitial fluid pressure, 
improved oxygenation and better penetration of drugs in the tumor, enhancing the efficacy of subsequent radiation and chemotherapy $[7,8]$. However, incorrect doses or scheduling of antiangiogenic agents might antagonize rather than augment the therapeutic response to chemotherapy. Therefore, optimal scheduling of treatment may be required to overcome the pharmacokinetic barriers and potentially result in longterm tumor remissions [9].

In the previous study, we designed and identified a new potent VEGFR2 inhibitor, SKLB1002, which showed dosedependent inhibitory activity in human umbilical vein endothelial cells and in human tumor xenografts in athymic mice with limited toxicity. Western blot analysis was conducted, which demonstrated that SKLB1002 inhibited VEGF-induced phosphorylation of VEGFR2 kinase and the downstream protein kinases including extracellular signal regulated kinase, focal adhesion kinase and Src [10]. In the present study, we investigated the effects of SKLB1002 on the determinants of vascular normalization, including changes in the morphology and function of tumor vasculature during the course of treatment. We found profound alterations in all of these parameters, which led us to investigate the effects of SKLB1002 on the subsequent response of tumors to chemotherapy. Finally, our studies showed for the first time that simultaneous treatment of chemotherapeutic agent and SKLB1002 significantly inhibited tumor growth and lung metastasis in immunocompetent animal models.

\section{Materials and Methods}

Animal and cell lines. BALB/c mice were purchased from the WestChinaExperimentalAnimalCenter. All experiments were carried out in accordance with the protocols approved by Animal Care and Use Committee of Sichuan University. Murine breast carcinoma cell line 4T1 and colon carcinoma cell line CT26 were purchased from the American Type Culture Collection.

Animal tumor model. BALB/c mice were inoculated s.c. with $5 \times 10^{5}$ of $4 \mathrm{~T} 1$ breast tumor cells. After 7 days, mice bearing tumors around $100 \mathrm{~mm}^{3}$ were selected and expanded for further study.

Immunofluorescence of CD31 and $a$-smooth muscle actin ( $\boldsymbol{\alpha}$-SMA). We used immunofluorescence to evaluate tumor vessel normalization by SKLB1002 treatment at different time points. 7 days after inoculation, mice bearing tumors were randomized into two groups. The dosing schedules were SKLB1002 $100 \mathrm{mg} / \mathrm{kg} / \mathrm{d}$ or vehicle once a day intraperitoneally. Fixed cryostat sections in each group were double-stained with the anti-CD31 (BD Biosciences) and anti- $\alpha$-SMA (Abcam) antibodies, as described previously [11]. Images were acquired using the fluorescence microscope equipped with a digital CCD camera (Olympus).

Detection of hypoxia with pimonidazole. To visualize hypoxic regions, mice were injected intravenously with $100 \mu \mathrm{l}$ of $10 \mathrm{mg} / \mathrm{ml}$ pimonidazole (Hypoxyprobe, Inc.) 15 minutes before sacrifice. Tumors were frozen for tissue sectioning and handled according to manufacturer's instructions. For each treatment group, the hypoxic areas were counted in six randomly captured images from three different tumors.

Electron microscopy. 5 days after initial administration of SKLB1002, tumor tissues in each group were fixed in 2.5\% glutaraldehyde and examined for vascular ultrastructure using the scanning electron microscope. Six tissue areas were analyzed and used for quantitative assessment of vessel phenotype in each group.

Intratumoral drug penetration. The effect of SKLB1002 on drug delivery and distribution was analyzed using highperformance liquid chromatography (HPLC) as described previously (12). 5 days after initial administration of SKLB1002, doxorubicin was given intravenously at a dose of $5 \mathrm{mg} / \mathrm{kg}$ to facilitate detection. 30 minutes after this bolus doxorubicin administration, whole blood and tumor tissue were collected. Prepared samples were subjected to the HPLC system consisting of a Waters 2695 separation module and a Waters 2996 photodiode array detector (Waters). Doxorubicin was detected by fluorescence, with emission wavelength at $254 \mathrm{~nm}$.

Tumor therapy studies. BALB/c mice were inoculated s.c. with $5 \times 10^{5}$ of $4 \mathrm{~T} 1$ cells or CT 26 cells, respectively. After 7 days, mice bearing $4 \mathrm{~T} 1$ tumors were randomized into four groups (16 per group). The dosing schedules were (1) vehicle; (2) SKLB1002 $100 \mathrm{mg} / \mathrm{kg} / \mathrm{d}$ intraperitoneally on day 1-5 of each week; (3) doxorubicin $5 \mathrm{mg} / \mathrm{kg}$ intravenously on day 6 of each week; (4) combined treatment. Meanwhile, mice bearing CT26 tumors were also randomized into four groups (16 per group):(1) vehicle; (2) SKLB1002 100 $\mathrm{mg} / \mathrm{kg} / \mathrm{d}$ intraperitoneally on day 1-5 of each week; (3) irinotecan $20 \mathrm{mg} / \mathrm{kg}$ intravenously on day 6 of each week; (4) combined treatment. The therapy was given every week for two cycles. The tumor volume was measured with a caliper every three days, and tumor volume was calculated using the following formula: tumor volume $\left(\mathrm{mm}^{3}\right)=\left[(\text { width })^{2}\right.$ $\times$ length] / 2.

28 days after tumor cell inoculation, 6 mice in each group were sacrificed, and the remaining mice (10 per group) were used for survival analysis. Tumors and lungs were excised, and metastatic nodules were observed under the dissecting microscope. Paraffin-embedded tumor sections were used to determine cellular proliferation in tumor tissues by examining PCNA expression. Meanwhile, to explore whether the treatment could effectively induce cell apoptosis in vivo, cell apoptosis in situ was determined using a TUNEL assay according to the manufacturer's instructions. For each group, the apoptotic index was counted in six randomly captured images from three different tumors.

Histopathologic toxicity studies. To investigate potential toxicity in mice during the treatment, we estimatedcontinuously associated indexes such as anorexia, weight loss and toxic deaths. The tissues of main organs were stained and sequentially detected by microscopic examination. 
Statistical analysis. Data were presented as mean $\pm \mathrm{SD}$. Data were analyzed statistically using one-way ANOVA. Survival curves were computed by the Kaplan-Meier method and compared by the log-rank test. $\mathrm{P}<0.05$ was considered statistically significant.

\section{Results}

SKLB1002 promotes vessel normalization and reduces tumor hypoxia. We examined the effect of SKLB1002 on tumor vessel density and pericyte coverage at 1-7 days after a daily dose of SKLB1002 to mice with established 4T1 tumor. Tumors in SKLB1002 group at each of these time points showed a significant and progressive decrease in tumor vessel density, as assessed by CD31, compared with control group.
Since vascular normalization by antiangiogenic therapy was expected to result in greater coverage of pericytes in basement membrane, we simultaneously stained for the pericyte marker a-SMA and found significantly increased pericyte coverage of tumor vessels over the time with treatment in SKLB1002 group (Fig. 1A, 1C and 1D). Hypoxic area in control tumors progressively increased, being most notable at 5 days. Interestingly, despite the obvious decrease in tumor vessel density, there was a less hypoxic area within sections of SKLB1002 treated tumors (Fig. 1B and 1E).

SKLB1002 normalizes the architecture and function of the tumor vasculature. To further examine morphologic changes of the tumor vasculature with SKLB1002 treatment, we used scanning electron microscopy for in vivo analysis. Microphotographs showed that fewer vessels in SKLB1002
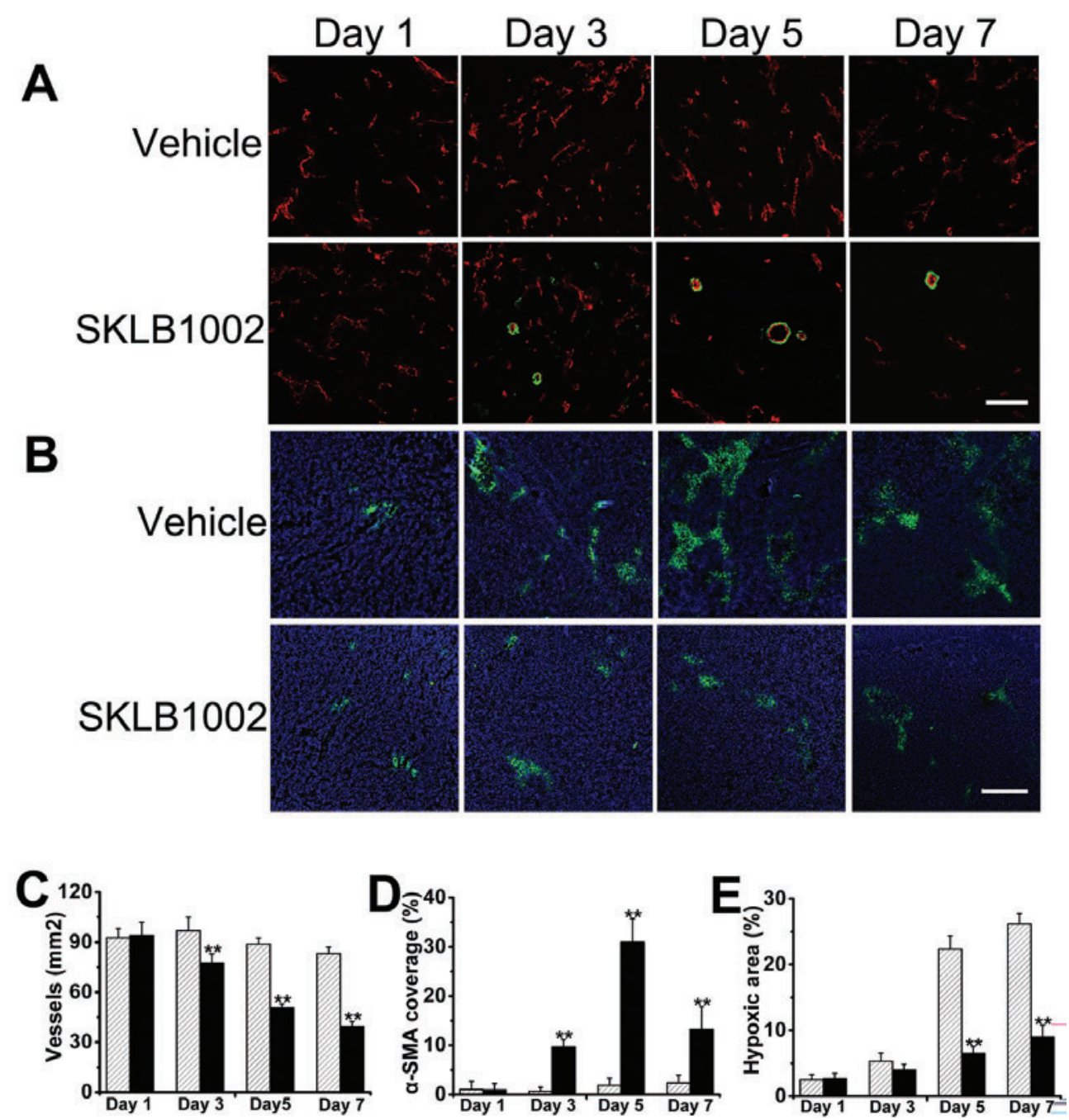

Figure 1. A, the effect of $100 \mathrm{mg} / \mathrm{kg} / \mathrm{d}$ SKLB1002 on the histology of tumor vessels within established 4T1 breast tumor. Representative CD31 (red color) and $\alpha$-SMA (green color) immunofluorescence was captured at day 1, 3, 5 and 7 after SKLB1002 administration. Scale bar, $100 \mu \mathrm{m}$. B, staining for PIMO indicated hypoxic regions in SKLB1002-treated and vehicle-treated tumors. Scale bar, $500 \mu \mathrm{m}$. C-E, the respective histograms revealing less vessels $(C$, $\mathrm{n}=6$; ${ }^{* *}, P<0.05$ at day 3,5 and 7$)$, more $\alpha$-SMA coverage $\left(\mathrm{D}, \mathrm{n}=6\right.$; ${ }^{* *}, P<0.05$ at day 3,5 and 7$)$ and smaller hypoxic area $\left(\mathrm{E}, \mathrm{n}=6\right.$; $^{* *}, P<0.05$ at day 5 and 7) within SKLB1002-treated (black columns) than vehicle-treated tumors (shadow columns). 
treated tumors had abnormal vessels, characterized by disconnected and multilamellar endothelial cells (Fig. 2A and 2B). We next investigated how these changes in tumor vessel structure would affect tumor penetration of systemically administered chemotherapeutic agents. After the bolus doxorubicin administration, HPLC analysis revealed a 2.2 fold increase of doxorubicin concentration in SKLB1002 treated 4T1 tumors compared with the control group. No significant change was observed in the plasma level of doxorubicin in the two groups, highlighting the improved penetration of doxorubicin following SKLB1002 treatment (Fig. 2C).

The synergic antitumor effects of SKLB1002 and chemotherapeutic agents. The structural normalization of tumor vasculature following SKLB1002 treatment prompted us to ask whether administration of a chemotherapeutic drug could enhance the anti-tumor response via enhanced drug delivery. The agent doxorubicin was the commonly used drug in breast cancer, meanwhile irinotecan was the commonly used drug in colon and rectum cancers. Monotherapy with doxorubicin inhibited 4T1 tumor growth and enhanced animal survival compared with controls. However, pre-treated with SKLB1002, doxorubicin injection resulted in a significant enhancement of the anti-tumor response compared with SKLB1002 or doxorubicin monotherapy (Fig. 3A). Median survival for the specific combination treatment group was 45 days versus 37 days for the animals that received SKLB1002 alone or 36 days for animals that received doxorubicin alone (Fig. 3C). Furthermore, we found similar results in another experiment
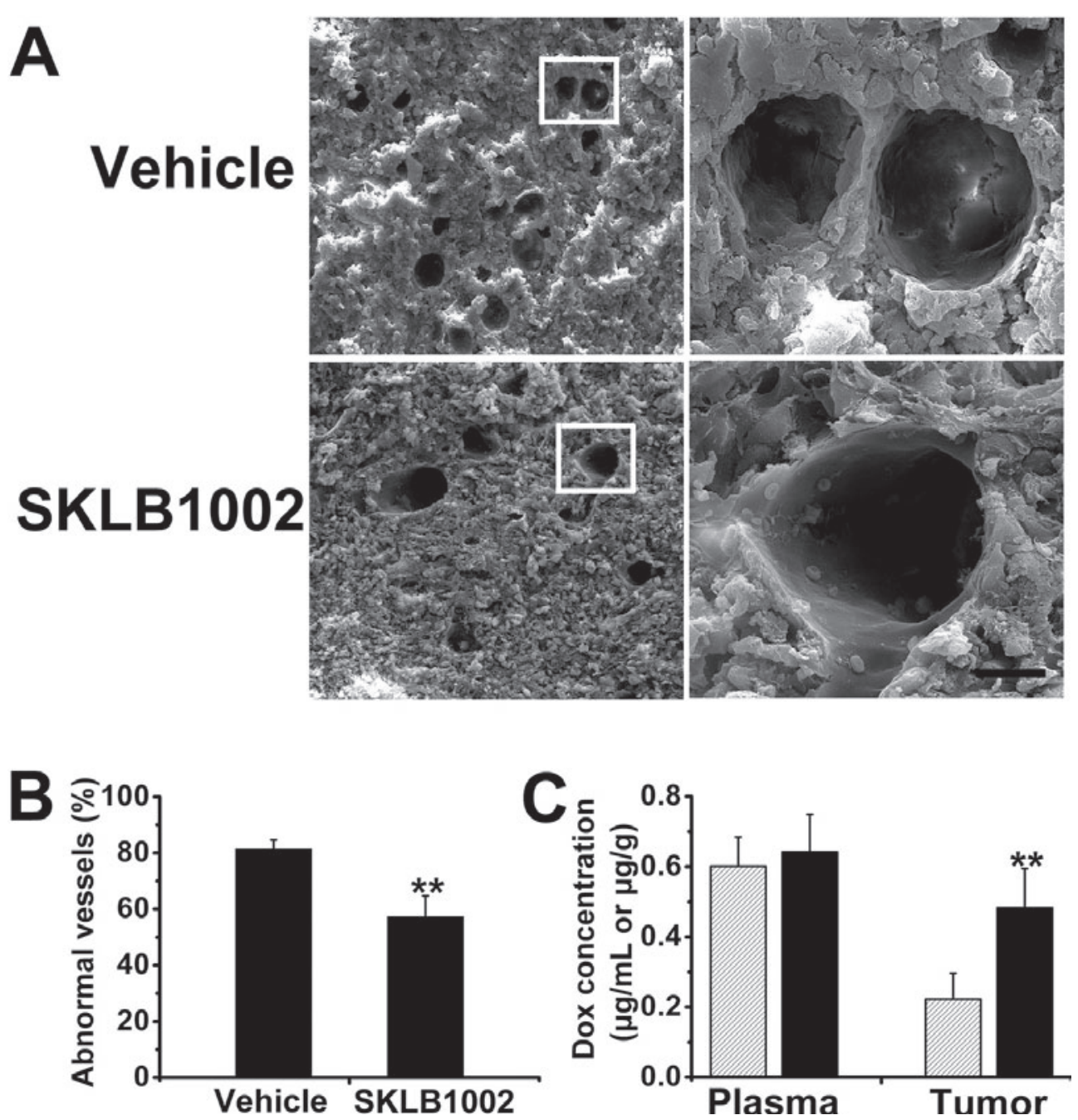

Figure 2. A and B, at day 5 after SKLB1002 administration, micrographs of scanning electron microscope showed abnormal tumor vessel with multilayers of disconnected endothelial cells in vehicle-treated tumors and normal vessel with compactly connected endothelial cells in SKLB1002-treated tumors. Scale bar, $10 \mu \mathrm{m}$. The histograms exhibited fraction of abnormal vessels $\left(n=6\right.$; $\left.{ }^{*}, P<0.05\right)$. C, HPLC-based determination of doxorubicin concentration in $4 \mathrm{~T} 1$ tumors and plasma, simultaneously. 2.2 fold increase in doxorubicin concentration was found in $4 \mathrm{~T} 1$ tumors with SKLB1002 pretreatment (black columns). No significant difference was found in plasma. 

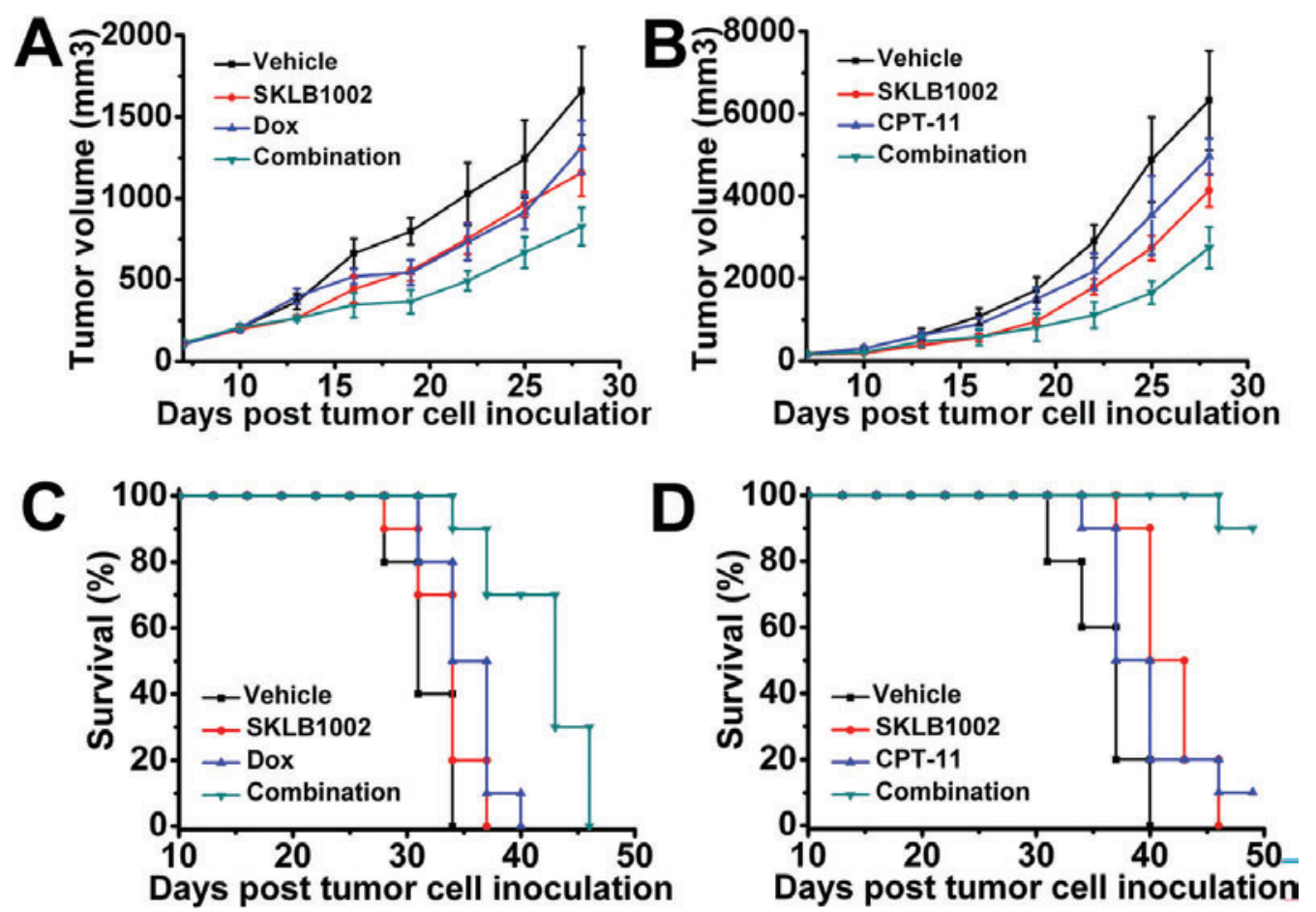

Figure 3. A and C, mice were subcutaneously implanted with 4T1 cells. After 7 days, mice were randomized into four groups: (1) vehicle; (2) SKLB1002 $100 \mathrm{mg} / \mathrm{kg} / \mathrm{d}$ daily intraperitoneally, day 1 -day 5 every week; (3) doxorubicin $5 \mathrm{mg} / \mathrm{kg}$ intravenously, day 6 every week; (4) combined treatment. The whole course of treatment was 2 weeks. The combined treatment group resulted in significant inhibition of tumor growth $(\mathrm{A}, \mathbf{n}=6$; ANOVA; $P<0.05)$ and had a longer survival period $(C, n=10 ; \log$-rank test; $P<0.05)$ as compared with other groups. $B$ and $D$, mice were subcutaneously implanted with CT26 cells. After 7 days, mice were randomized into four groups: (1) vehicle; (2) SKLB1002 $100 \mathrm{mg} / \mathrm{kg} / \mathrm{d}$ daily intraperitoneally, day 1 -day 5 every week; (3) irinotecan $20 \mathrm{mg} / \mathrm{kg}$ intravenously, day 6 every week; (4) combined treatment. The whole course of treatment was 2 weeks. The combined treatment group resulted in significant inhibition of tumor growth $(\mathrm{B}, \mathrm{n}=6$; ANOVA; $P<0.05)$ and had a longer survival period $(\mathrm{D}$, $\mathrm{n}=10$; log-rank test; $P<0.05)$ as compared with other groups.

in mice bearing CT26 colon carcinoma using irinotecan (Fig. $3 \mathrm{~B}$ and $3 \mathrm{D})$.

To identify the mechanisms underlying tumor growth suppression, we investigated tumor proliferation and apoptosis after multiple treatments. As shown in Fig. 4A and 4C, tumor tissues of mice treated with SKLB1002 and doxorubicin exhibited much less staining for PCNA, compared with that in tumor tissues of other groups. Additionally, TUNEL assay showed a significantly greater percentage of apoptotic cells in the combined treated tumors in relation to the other tumors (Fig. 4B and 4D). Thus, we concluded tumor growth suppression was caused, at least partially, by enhanced tumor cell apoptosis and reduced proliferation.

4T1 breast tumor had highly metastatic potential, and metastatic cells could be detected in various organs, especially the lung. Therefore, it was important to evaluate the effect of combined therapy not only on local tumor but also on the formation of distant metastases. Our examination of the whole lungs revealed that the number of the metastatic colonies was much lower in combined treatment group than that in other groups as observed by light microscopy (Supplementary Fig. $5 \mathrm{~A}$ and $5 \mathrm{~B})$.
The combined therapy is not toxic at therapeutically active doses. No significant differences in weights were found among four groups, and toxic pathologic changes in main organs especially the heart and small intestine were not detected by microscopic examination (Supplementary Fig. 5C, $5 \mathrm{D}$ and $5 \mathrm{E})$.

\section{Discussion}

The concept of vascular normalization relied primarily on the idea of targeting the tumor vasculature via blocking VEGF signaling, providing an opportunity for enhanced efficacy of radiation and chemotherapy. However, extensive destruction of tumor vessels by antiangiogenic therapy could provoke vascular regression and ultimately hinder the delivery of oxygen and drugs. To find an optimum combination therapy, we must reveal the mechanism of antiangiogenic agents and use this theory to optimize the therapeutic scheduling [13-15]. Here we continuously monitored the time course of molecular changes in the vasculature of 4T1 murine breast tumor in response to treatment with SKLB1002. We observed that 3 days after administration, pericyte coverage was significantly 

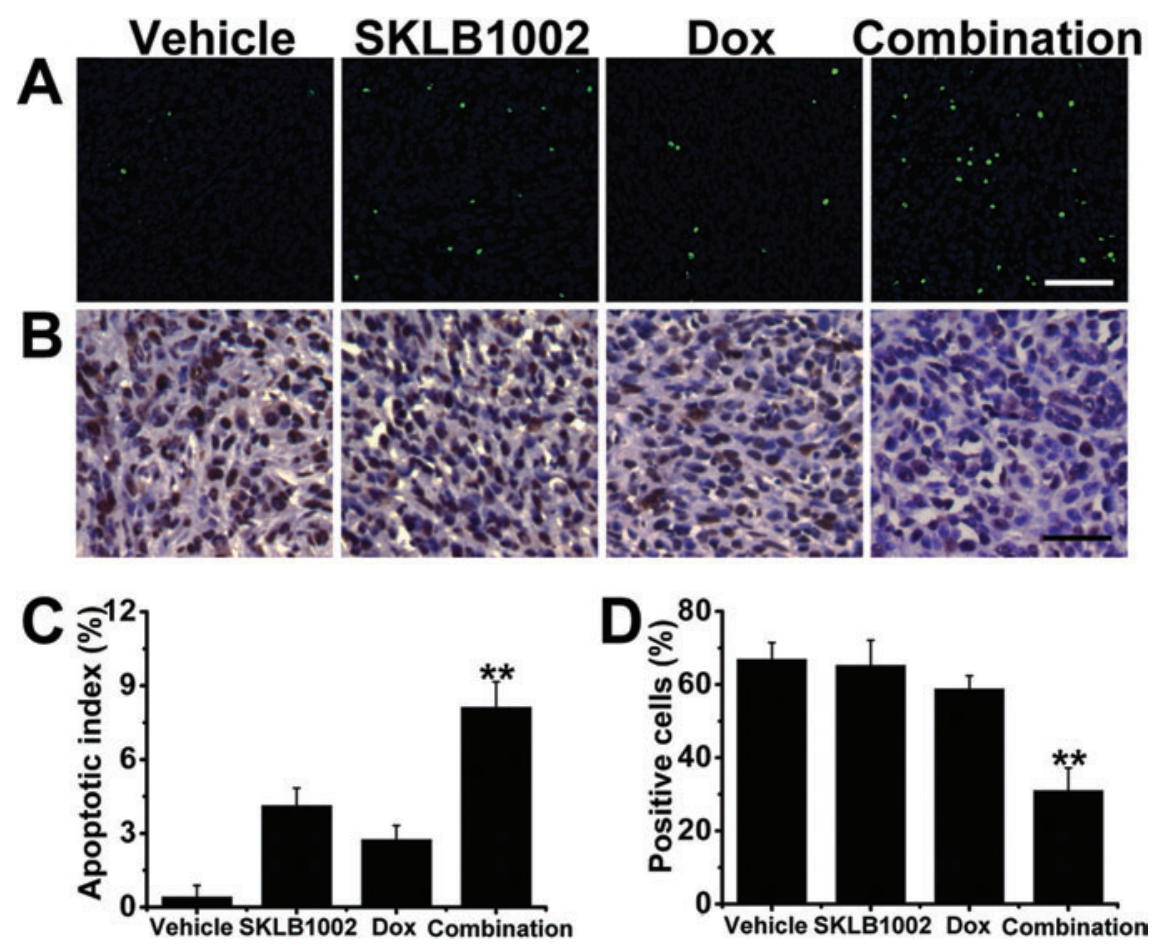

Figure 4. after mice were sacrificed, the tumor samples were taken out and paraffin-embedded sections were prepared for TUNEL analysis and immunohistochemistry. A, TUNEL analysis showed in situ cell apoptosis of 4T1 tumor. Size bar, $100 \mu \mathrm{m}$. B, Immunohistochemistry showed the level of PCNA expression, which correlated cell proliferation in tumor tissue. Size bar, $50 \mu \mathrm{m}$. C and D, the combined treatment induced cell apoptosis $\left(C, \mathbf{n}=6\right.$; ${ }^{* *}, P<$ $0.05)$ and reduced level of PCNA expression $\left(D, \mathbf{n}=6 ;{ }^{* *}, P<0.05\right)$ in vivo, which correlated significant inhibition of $4 \mathrm{~T} 1$ tumor growth.
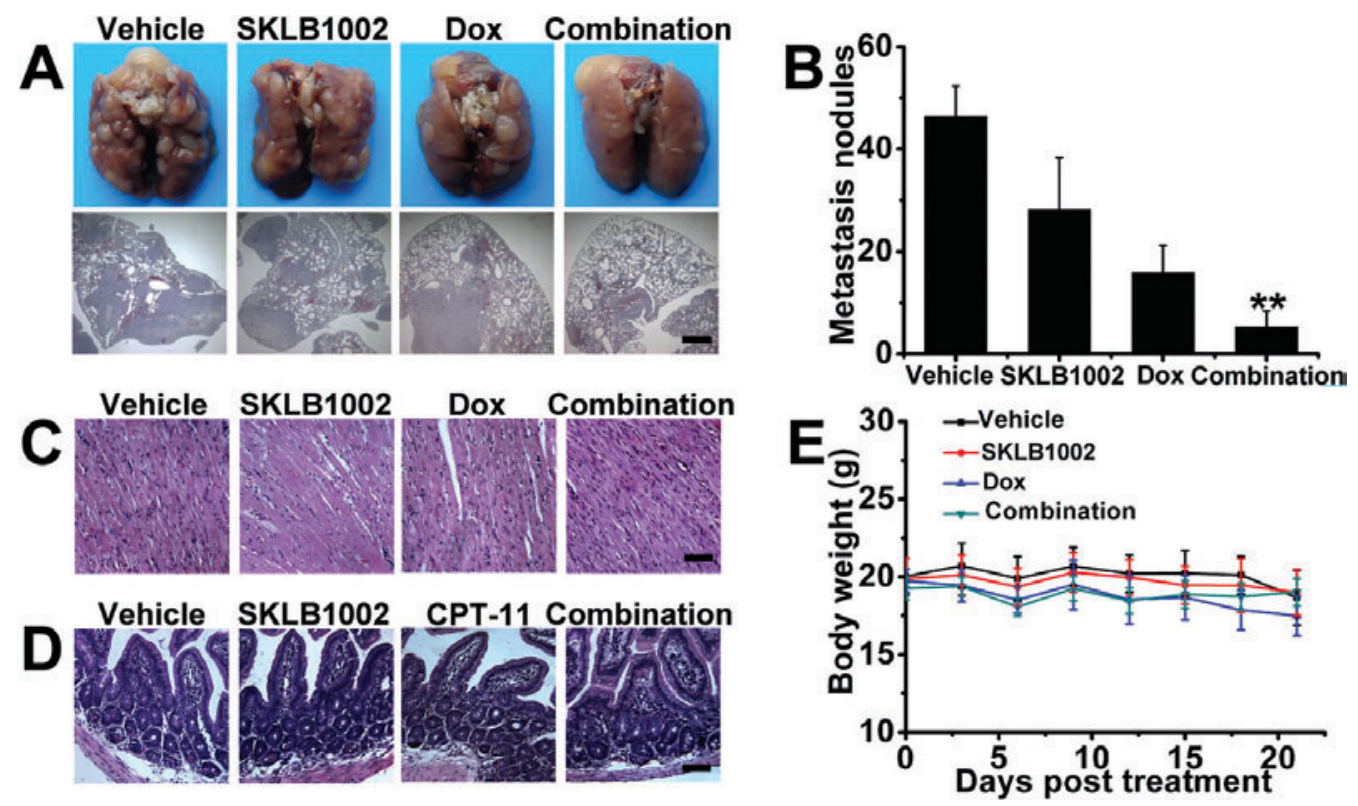

Figure 5. A, after the termination of in vivo experiments, lungs of mice in each group were excised. Metastatic nodule was observed under the dissecting microscope and subsequently evaluated by H\&E staining. B, SKLB1002 in combination with doxorubicin had more profound inhibitory effects on $4 \mathrm{~T} 1$ breast tumor metastasis formation than each therapy applied separately $\left(\mathrm{n}=6\right.$; $\left.{ }^{* *}, P<0.05\right)$. C, SKLB1002 did not increase doxorubicin-induced cardiotoxicity. H\&E staining of paraffin-embedded sections of the heart in each group did not exhibit obvious pathologic abnormalities. D, SKLB1002 did not increase risk of irinotecan-associated toxicity. H\&E staining of paraffin-embedded sections of the small intestine in each group did not exhibit obvious pathologic abnormalities. E, body weight of tumor-bearing mice was plotted at 2-day intervals. The curve of combined treatment group paralleled very closely to that of the other groups with no significant differences among them $(\mathbf{n}=6 ; P>0.05)$. 
improved in mice receiving SKLB1002. However, the process seemed to be transient, reaching peak at day 5 and decreasing at day 7 . When pericyte coverage reached its peak at day 5 in the treated mice, we also found a significant decrease in the density of tumor vessels, consistent with the previous study [16]. Despite causing an overall decrease in intratumoral vessel density, SKLB1002 resulted in more efficient oxygenation of tumors in mice with established tumor model. To further explain the paradox, we used scanning electron microscope for in vivo analysis of ultrastructure in tumor vessel with SKLB1002 treatment. These images showed dramatic quantitative differences in tumor vessel architecture after treatment, which had fewer vessels containing abnormal multilayers of disconnected endothelial cells. Subsequently we estimated how these alterations in tumor vessel structure would affect tumor penetration of systemically administered chemotherapeutic agents. Several studies indicated that the consequence of remodeling the intratumoral vasculature was an associated improvement in intratumoral delivery of systemic chemotherapy. Consistent with previous studies $[17,18]$, HPLC analysis revealed a 2.2 fold increase in doxorubicin concentration in SKLB1002 pretreated tumors, and no significant change of doxorubicin was observed in the plasma in both groups of mice.

Clinical studies using VEGF signaling inhibitors had shown only modest clinical activity when used as single agents. Previous data supported a proposition that two modes of resistance underlay such results: intrinsic non-responsiveness and adaptive resistance [5]. Intrinsic non-responsiveness was the absence of any beneficial effect of an antiangiogenic therapy. By contrast, after an initial response phase, adaptive resistance referred to the ability of tumors to evade the therapeutic blockade by inducing other signaling of neovascularization. Then the second wave of angiogenesis initiated by the residual tumor cells could happen, leading to the later relapse of tumors $[19,20]$. To impede the insidious consequence of single antiangiogenic therapy, concomitant chemotherapy was needed to target the tumor cells directly and prevent tumor recurrence.

Doxorubicin was an anthracycline drug widely used in chemotherapy regimen for patients with breast cancer. Despite its excellent clinical results, doxorubicin had a relatively low therapeutic index and its clinical application was greatly limited due to cumulative dose-related cardiotoxicity and common side effects such as myelosuppression [21]. Therefore, combination treatment with another highly effective non-toxic drug which could lower the dose of doxorubicin would be desirable.

The structural normalization of residual tumor vasculature following SKLB1002 treatment prompted us to ask whether administration of the chemotherapeutics in that time-period would enhance the overall anti-tumor response via greater accessibility of the drug to tumor cells. Our data were in agreement with accumulating evidence that the tumor microenvironment indeed affected tumor drug delivery and therapeutic effects $[15,22]$. Finally, compared with the vehicle control, SKLB1002 combined with doxorubicin reached more than $50 \%$ inhibition against $4 \mathrm{~T} 1$ tumor models in $\mathrm{BALB} / \mathrm{c}$ mice. One potential explanation for the effect was that the structural normalization of tumor vasculature by SKLB1002 treatment resulted in increased efficiency of the vessels in delivering the chemotherapeutics to the tumor cells, thereby leading to synergy. However, it was uncertain whether SKLB1002 would achieve the same synergic effects with different chemotherapeutic agent against different types of solid tumors. We subsequently found similar results using CT26 colon tumor model and another chemotherapeutic agent, irinotecan.

Untreatable metastasis was the main cause of mortality in patients with cancer. Hypoxia was clinically associated with metastasis and poor patient outcome, for it induced genetic instability and selected for more malignant cells with increased metastatic potential $[23,24]$. Our findings suggested that SKLB1002 inhibited metastasis in part by altering vessel morphology. These changes, including an increased pericyte coverage and better oxygenation, rendered cancer cells less invasive and prevented the shedding of cancer cells into the circulation, which were early steps necessary for metastasis.

Our findings highlight an integrative approach to cancer therapy wherein SKLB1002 therapy is precisely scheduled with chemotherapy to maximize the synergistic effects against tumors.

Acknowledgements: This study was funded by the National Key Basic Research Program (973 Program) of China (2010CB529900).

\section{References}

[1] SIEGEL R, WARD E, BRAWLEY O, JEMAL A. Cancer statistics, 2011: the impact of eliminating socioeconomic and racial disparities on premature cancer deaths. CA Cancer J Clin 2011; 61: 212-236. http://dx.doi.org/10.3322/caac.20121

[2] HANAHAN D and WEINBERG RA. Hallmarks of cancer: the next generation. Cell 2011; 144: 646-674. http://dx.doi. org/10.1016/j.cell.2011.02.013

[3] KERBEL RS. Tumor angiogenesis. N Engl J Med 2008; 358 : 2039-2049. http://dx.doi.org/10.1056/NEJMra0706596

[4] BICKNELL R and HARRIS AL. Anticancer strategies involving the vasculature: vascular targeting and the inhibition of angiogenesis. Semin Cancer Biol 1992; 3: 399-407.

[5] BERGERS G AND HANAHAN D. Modes of resistance to anti-angiogenic therapy. Nat Rev Cancer 2008; 8: 592-603. http://dx.doi.org/10.1038/nrc2442

[6] HURWITZ H, FEHRENBACHER L, NOVOTNY W, CARTWRIGHT T, HAINSWORTH J et al. Bevacizumab plus irinotecan, fluorouracil, and leucovorin for metastatic colorectal cancer. N Engl J Med 2004; 350: 2335-2342. http://dx.doi. org/10.1056/NEJMoa032691

[7] JAIN RK. Normalization of Tumor Vasculature: An Emerging Concept in Antiangiogenic Therapy. Science 2005; 307: 58-62. http://dx.doi.org/10.1126/science.1104819

[8] ESCORCIA FE, HENKE E, MCDEVITT MR, VILLA CH, SMITH-JONES P et al. Selective killing of tumor neovas- 
culature paradoxically improves chemotherapy delivery to tumors. Cancer Res 2010; 70: 9277-9286. http://dx.doi. org/10.1158/0008-5472.CAN-10-2029

[9] MAITY A AND BERNHARD EJ. Modulating tumor vasculature through signaling inhibition to improve cytotoxic therapy. Cancer Res 2010; 70: 2141-2145. http://dx.doi.org/ 10.1158/0008-5472.CAN-09-3615

[10] ZHANG S, CAO Z, TIAN H, SHEN G, MA Y et al. SKLB1002, a novel potent inhibitor of VEGF receptor 2 signaling, inhibits angiogenesis and tumor growth in vivo. Clin Cancer Res 2011; 17: 4439-4450. http://dx.doi.org/10.1158/1078-0432.CCR-10$\underline{3109}$

[11] PIROT N, DELEUZE V, EL-HAJJ R, DOHET C, SABLITZKY $\mathrm{F}$ et al. LYL1 activity is required for the maturation of newly formed blood vessels in adulthood. Blood 2010; 115: 52705279. http://dx.doi.org/10.1182/blood-2010-03-275651

[12] BHATTACHARYA A, SESHADRI M, OVEN SD, TOTH K, VAUGHAN MM et al. Tumor vascular maturation and improved drug delivery induced by methylselenocysteine leads to therapeutic synergy with anticancer drugs. Clin. Cancer Res 2008; 14: 3926-3932. http://dx.doi.org/10.1158/10780432.CCR-08-0212

[13] WINKLER F, KOZIN SV, TONG RT, CHAE SS, BOOTH MF et al. Kinetics of vascular normalization by VEGFR2 blockade governs brain tumor response to radiation: role of oxygenation, angiopoietin-1, and matrix metalloproteinases. Cancer Cell 2004; 6: 553-563.

[14] SATO Y. Persistent vascular normalization as an alternative goal of anti-angiogenic cancer therapy. Cancer Sci 2011; 102: 1253-1256. http://dx.doi.org/10.1111/j.1349-7006.2011.01929.x

[15] DICKSON PV, HAMNER JB, SIMS TL, FRAGA CH, NG $\mathrm{CY}$ et al. Bevacizumab-induced transient remodeling of the vasculature in neuroblastoma xenografts results in improved delivery and efficacy of systemically administered chemotherapy. Clin Cancer Res 2007; 13: 3942-3950. http://dx.doi. org/10.1158/1078-0432.CCR-07-0278

[16] TONG RT, BOUCHER Y, KOZIN SV, WINKLER F, HICKLIN DJ et al. Vascular normalization by vascular endothelial growth factor receptor 2 blockade induces a pressure gradi- ent across the vasculature and improves drug penetration in tumors. Cancer Res 2004; 64: 3731-3736. http://dx.doi. org/10.1158/0008-5472.CAN-04-0074

[17] CERNIGLIA GJ, PORE N, TSAI JH, SCHULTZ S, MICK R et al. Epidermal growth factor receptor inhibition modulates the microenvironment by vascular normalization to improve chemotherapy and radiotherapy efficacy. PLoS ONE 2009; 4: e6539. http://dx.doi.org/10.1371/journal.pone.0006539

[18] QAYUM N, IM J, STRATFORD MR, BERNHARD EJ, MCKENNA WGet al. Modulation of the tumor microvasculature by phosphoinositide-3 kinase inhibition increases Doxorubicin delivery in vivo. Clin Cancer Res 2012; 18: 161-169. http:// dx.doi.org/10.1158/1078-0432.CCR-11-1413

[19] PAEZ-RIBES M, ALLEN E, HUDOCK J, TAKEDA T, OKUYAMA $\mathrm{H}$ et al. Antiangiogenic therapy elicits malignant progression of tumors to increased local invasion and distant metastasis. Cancer Cell 2009; 15: 220-231. http://dx.doi. org/10.1016/j.ccr.2009.01.027

[20] EBOS JM, LEE CR, CRUZ-MUNOZ W, BJARNASON GA, CHRISTENSEN JG et al. Accelerated metastasis after shortterm treatment with a potent inhibitor of tumor angiogenesis. Cancer Cell 2009; 15: 232-239. http://dx.doi.org/10.1016/ j.ccr.2009.01.021

[21] VAN LUMMEL M, VAN BLITTERSWIJK WJ, VINK SR, VELDMAN RJ, VAN DER VALK MA et al. Enriching lipid nanovesicles with short-chain glucosylceramide improves doxorubicin delivery and efficacy in solid tumors. FASEB J 2011; 25: 280-289. http://dx.doi.org/10.1096/fj.10-163709

[22] SINGH JAGGI J, HENKE E, SESHAN SV, KAPPEL BJ, CHATTOPADHYAY Det al. Selective alpha-particle mediated depletion of tumor vasculature with vascular normalization. PLoS ONE 2007; 2: e267. http://dx.doi.org/10.1371/journal. pone.0000267

[23] SEMENZA GL. Targeting HIF-1 for cancer therapy. Nat Rev Cancer 2003; 3: 721-732. http://dx.doi.org/10.1038/nrc1187

[24] ERLER JT, BENNEWITH KL, NICOLAU M, DORNHOFER $\mathrm{N}, \mathrm{KONG} \mathrm{C}$ et al. Lysyl oxidase is essential for hypoxia-induced metastasis. Nature 2006; 440: 1222-1226. http://dx.doi. $\underline{\text { org/10.1038/nature } 04695}$ 